\title{
Structural analysis of testicular appendices in patients with cryptorchidism
}

\author{
Guilherme D. Tostes, Suelen F. Costa, João P. de Carvalho, Waldemar S. Costa, Francisco J.B. \\ Sampaio, Luciano A. Favorito
}

Urogenital Research Unit, Rio de Janeiro State University, Rio de Janeiro, Brazil

\section{ABSTRACT}

Objectives: Report the incidence and structure of testicular appendices (TAs) in patients with cryptorchidism, comparing their incidence with epididymal anomalies (EA) and patency of the vaginal process (PVP) and analyzes the structure of TAs.

Material and Methods: We studied 72 testes of patients with cryptorchidism (average of 6 years), and 8 testes from patients with hydroceles (average of 9 years). We analyzed the relations among the testis, epididymis and PVP and prevalence and histology of the TAs. The appendices of 10 patients with cryptorchidism and 8 with hydrocele were dissected and embedded in paraffin and stained with Masson trichrome; Weigert and Picro-Sirius Red with polarization and immunohistochemistry analysis of the collagen type III fibers to observe collagen. The stereological analysis was done with the software Image Pro and Image $\mathrm{J}$, using a grid to determine volumetric densities ( $\mathrm{Vv}$ ). Means were statistically compared using the ANOVA and unpaired T test $(\mathrm{p}<0.05)$.

Results: Of the 72 testes with cryptorchidism, 20 (27.77\%) presented EA, 41 (56.9\%) had PVP and $44(61.1 \%)$ had TAs. Of the 44 testes with cryptorchidism and appendices, $30(68.18 \%)$ presented PVP and 11 (25\%) presented EA. There was no alteration of the epithelium in the appendices of patients in both groups. Stereological analysis documented the prevalence of ESFs (mean of 1.48\%), prevalence of veins (mean of 10.11\%) and decrease $(p=0.14)$ of SMCs in the TAs of patients with cryptorchidism (mean $=4.93 \%$ ). Collagen III prevailed in the TAs of patients with cryptorchidism.

Conclusion: The testicular appendices presented significant structural alteration in the patients with cryptorchidism, indicating that TAs present a structural remodeling.

\section{ARTICLE INFO}

\section{Key words:}

Cryptorchidism; anatomy and histology; Epididymis; abnormalities

Int Braz J Urol. 2013; 39: 240-7

Submitted for publication: October 03, 2012

Accepted after revision: January 10, 2013

\section{INTRODUCTION}

Testicular and epididymal appendices have been considered congenital anomalies (1). The appendix testis or hydatid of Morgagni is believed to be the embryologic remnant of the cranial end of the Mullerian or paramesonephric duct (2). It is present in more than $90 \%$ of males, it varies in size from 1-10 $\mathrm{mm}$ in diameter and it is usually pedunculated, which predisposes to torsion. It is the most frequently twisted of the four testicular appendages (3-5). The other are the appendix of the epididymis, which is a remnant of the wolffian duct, the paradidymis and the vas aberrant. These vestigial structures have a similar histology, composed of gelatinous and vascular connective tissue covered with a columnar epithelium (6).

The functions of testicular appendices are controversial, but they may control the amount of serous fluid within the space of the vaginal 
process (7). Another hypothesis suggests that the surface epithelium, subepithelial capillaries and lymphatic vessels of testicular appendices form a functional unit (8). Some studies have analyzed the embryology (9) and structure of the testicular and epididymal appendices $(3,10)$.

Studies of the incidence of these structures in patients with cryptorchidism and comparison with individuals without testicular position anomalies are rare in the literature (11). There are no published studies analyzing the structure of testicular appendices in patients with cryptorchidism.

This work reports the incidence and structure of testicular appendices (TAs) in patients with cryptorchidism, comparing their incidence with epididymal anomalies and patency of the vaginal process and analyzes the structure of testicular appendices to assess whether their architecture is altered when cryptorchidism is present.

\section{MATERIALS AND METHODS}

The present work received institutional review committee and parent approval and has been carried out in accordance to the ethical standards of the responsible institutional committee on human experimentation.

We analyzed 72 testes from 55 patients with cryptorchidism (17 with bilateral cryptorchidism) and compared them with a control group of 8 testes from patients with hydroceles. The patients with cryptorchidism had ages from 1 to 13 years (average of 6 years), while those in the control group ranged from 1 to 14 years old (average of 9 years). In the two groups, we analyzed the relations among the testis, epididymis and patency of the vaginal process and the incidence and histology of the testicular appendices.

To analyze the relations between the testis and epididymis during surgery, we used a previous classification $(12,13)$. By this classification, there are six types of relations between the testis and epididymis: Type I - epididymis attached to the testis at the head and tail; Type II - epididymis totally attached to the testis; Type III - epididymis attached to the testis only at the head; Type IV epididymis attached to the testis only at the tail; Type V - no visible connection between the testis and epididymis; and Type VI - epididymal atresia. Type I and II relations are considered normal while the other types are considered to be anatomical anomalies (12). To analyze the vaginal process we considered two situations: (a) complete obliteration of the vaginal process between the internal inguinal ring and the superior pole of the testis; and (b) complete patency of the vaginal process.

In relation to the testicular appendices, we analyzed the following situations in the two groups during the surgeries: I) absence of testicular and epididymal appendices; II) presence of testicular appendix only; III) presence of epididymal appendix only; IV) presence of testicular and epididymal appendices; V) presence of two epididymal appendices and one testicular appendix; and VI) presence of paradidymis or vas aberrans of Haller.

The appendices of 10 patients with cryptorchidism and 8 patients with hydrocele were separated from the other structures and fixed in $10 \%$ buffered formalin, and routinely processed for paraffin embedding. Then $5 \mu \mathrm{m}$ thick sections were obtained at $200 \mu \mathrm{m}$ - intervals. Smooth muscle cells, connective tissue and elastic system fibers were studied by histochemical and immunolabeling methods.

The sections were stained with hematoxylin-eosin to assess the integrity of the tissue. We performed the following stainings: Masson's trichrome, in order to quantify connective tissue and smooth muscle, and Weigert resorcin fucsin with previous oxidation in order to observe elastic system fibers. Connective tissue, smooth muscle cells and elastic system fibers were quantified by a digital method (14). Five sections per specimen were stained, and five fields of each section were selected. All selected fields were photographed with an Olympus DP70 camera coupled to an Olympus BX51 microscope. The images were processed and saved with the Image Pro software. The tissue was quantified using the Image J software to determine the area density (Ad) of each component (14). Immunolabeling was performed to confirm the results. For smooth muscle cells (SMCs), Monoclonal Alfa Actin Antibody 08-0106 (Zymed Laboratories, San Francisco, CA, United States) was used. To confirm the results for elastic 
fibers (EF), Monoclonal Elastin Antibody ab 9519 (Abcam Laboratories, Cambridge, MA, United States) was used.

Means were statistically compared using the unpaired t-test and linear regression was performed when applicable. Statistical relevance was accepted if $\mathrm{p}<0.05$.

\section{RESULTS}

Of the 72 testes with cryptorchidism, 7 were abdominal (9.7\%), 54 (75\%) were located in the inguinal canal and 11 (15.3\%) were supra-scrotal. Of the testis with cryptorchidism, 20 (27.77\%) presented epididymal anomalies, 41 (56.9\%) had patent vaginal process and 44 (61.1\%) had testicular appendices. The prevalence of the testicular appendices in the testis with cryptorchidism and their relation with the testicular position are reported in Table-1. There was no statistically significant difference in relation to the location of the appendices $(p=0.0652)$.

Of the 7 abdominal testes, all had patent vaginal process and 4 (57\%) had testicular appendices. Of the 54 testes located in the canal, 31 (57\%) had appendices, and of these, 21 (38.8\%) presented patent vaginal process and 19 (35.8\%) had epididymal anomalies. Of the 11 cases of supra-scrotal testes, 9 (82\%) had appendices, 5 $(55.5 \%)$ had patency of the vaginal process and 1 (11.1\%) presented epididymal anomaly. Finally, of the 8 cases in the control group, 7 had appendices
(87.5\%), 1 (14.28\%) had epididymal anomaly and $1(14.28 \%)$ had patent vaginal process.

Of the 44 testes with cryptorchidism and appendices, $30(68.18 \%)$ presented patent vaginal process and 11 (25\%) showed epididymal anomalies. Of the 28 testes with cryptorchidism and without appendices, 11 (39.28\%) presented patent vaginal process and 9 (32.14\%) showed epididymal anomalies. The relation between the type of testicular appendix found and the patency of the vaginal process and the presence of epididymal anomalies is shown in Table-2. There was no statistically significant difference in relation to the patency of vaginal process between the patients with cryptorchidism and appendices with the control group ( $\mathrm{p}$ $=0.053$ ) and with the patients with cryptorchidism without appendices $(p=0.252)$. Also there was no statistically significant difference in relation to the epididimal anomalies between the patients with cryptorchidism and appendices with the control group ( $p=0.305)$ and with the patients with cryptorchidism without appendices $(p=0.6830)$. Of the 55 patients, 17 had bilateral cryptorchidism, for a total of 34 testes. Of these, 1 was abdominal, 24 were located in the canal and 9 were supra-scrotal. Of the 34 testes of this group, 25 (73.5\%) had testicular appendices, with 13 being right testes and 12 left ones, with no statistical difference.

Sections stained with Masson's trichrome from the testicular appendix demonstrated a vascular stroma lined with pseudocolumnar epithelium. The stroma consisted of loose connective tissue

Table 1 - Incidence of the testicular and epididymal appendices studied with respect to position of the testes with cryptorchidism and the testes in the control group. There was no statistically significant difference in relation to the location of the appendices $(\mathrm{p}=\mathbf{0 . 0 6 5 2})$.

\begin{tabular}{lcccc}
\hline Appendices & Abdomen & Canal & Supra-Scrotal & Control \\
\hline No appendix & 3 & 23 & 2 & 1 \\
Testicular appendix & 1 & 23 & 7 & 5 \\
Epididymal appendix & 2 & 4 & 0 & 1 \\
Testicular and epididymal appendix & 1 & 4 & 2 & 1 \\
\hline Total & $\mathbf{7}$ & $\mathbf{5 4}$ & $\mathbf{1 1}$ & $\mathbf{8}$ \\
\hline
\end{tabular}


Table 2 - Relation between the type of appendix found and the patency of the vaginal process and presence of epididymal anomaly. There was no statistically significant difference in relation to the patency of vaginal process $(p=0.252)$ and epididymal anomalies $(p=0.6830)$ between the patients with cryptorchidism and appendices with the patients with cryptorchidism without appendices.

\begin{tabular}{lcc}
\hline Appendices & Patent vaginal process & Epididymal anomaly \\
\hline Testicular (31) & 20 & 5 \\
Epididymal (6) & 5 & 4 \\
Testicular and Epididymal (7) & 5 & 2 \\
\hline Total (44-100\%) & $\mathbf{3 0}(\mathbf{6 8 . 1 8 \% )}$ & $\mathbf{1 1 ( 2 5 \% )}$ \\
\hline
\end{tabular}

containing blood vessels, fibroblasts and varying numbers of acini (gland-like structures), which were lined with a non-ciliated columnar epithelium (Figure-1A). The epididymal appendices were vesicular structures. The cavity of the vesicle was lined with a pseudostratified columnar epithelium (Figure-1B). There was no apparent alteration between the epithelium in the appendices of patients with cryptorchidism and in the control group, as seen in Figures $1 \mathrm{~A}$ and $1 \mathrm{C}$.

Figure 1 - Appendix epithelium. A) Patient with cryptorchidism with 3-years-old, showing the vascular stroma lined with pseudostratified epithelium of the testicular appendix. Masson's trichrome x200. B) Patient of the control group with 13-year-old, showing epididymal appendix with cavitation covered by pseudostratified epithelium. Masson's trichrome $x 200$. C) Patient of the control group with 13-years-old, showing pseudostratified epithelium of the entire testicular appendix. Masson's trichrome x200.

A

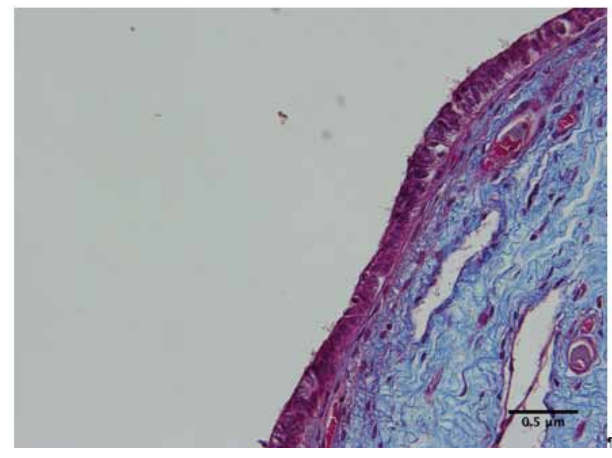

B

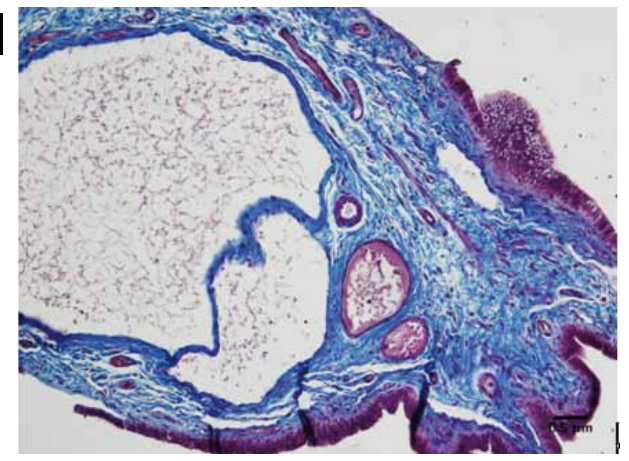

c

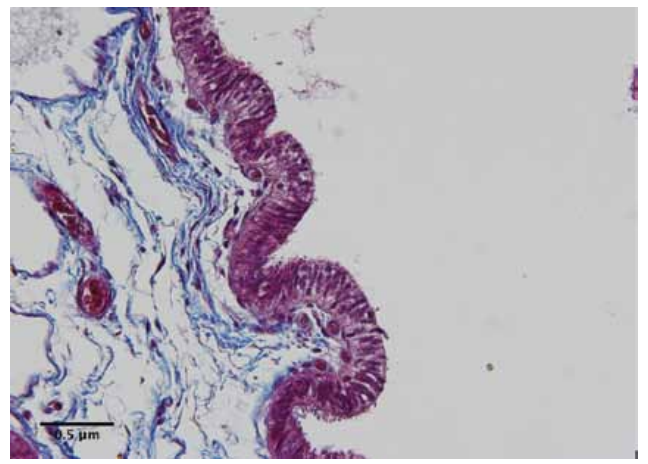


Stereological analysis documented a prevalence of elastic fibers in the testicular appendices of the patients with cryptorchidism (mean of $1.48 \%$ ) in comparison to the appendices of the patients with a hydrocele testis (mean of $0.29 \%$ ), a result that was not statistically different $(\mathrm{p}=$ 0.22 ). Figure- 2 shows the testicular appendices in patients with cryptorchidism and in the control group stained by Weigert's resorcin-fucsin with oxidation. The testes of the patients with cryptorchidism contain a larger quantity of elastic fibers.

Stereological analysis also showed a greater incidence of vessels in the testicular appendi- ces of the patients with cryptorchidism (mean of $10.11 \%$, standard deviation of 6.88 ) in relation to the control group (mean of $4.77 \%$, standard deviation of 1.9). This difference was statistically significant ( $p<0.001)$. Figure-3 shows the testicular appendices in patients with cryptorchidism and those of the control group, stained by Masson's trichrome. A greater number of vessels can be observed in the testes of patients with cryptorchidism.

Stereology analysis showed a decrease without significance $(p=0.14)$ of smooth muscle cells in the appendices of patients with cryptor-

Figure 2 - Elastic system fibers of the testicular appendix. A) Testicular appendix of 13-year-old patient with cryptorchidism. Weigert x400. B) Testicular appendix of 13-year-old patient of the control group. Weigert $\mathbf{4 0 0}$. Note the marked increase of elastic fibers (brown) in the patient with cryptorchidism when compared to the control.

A

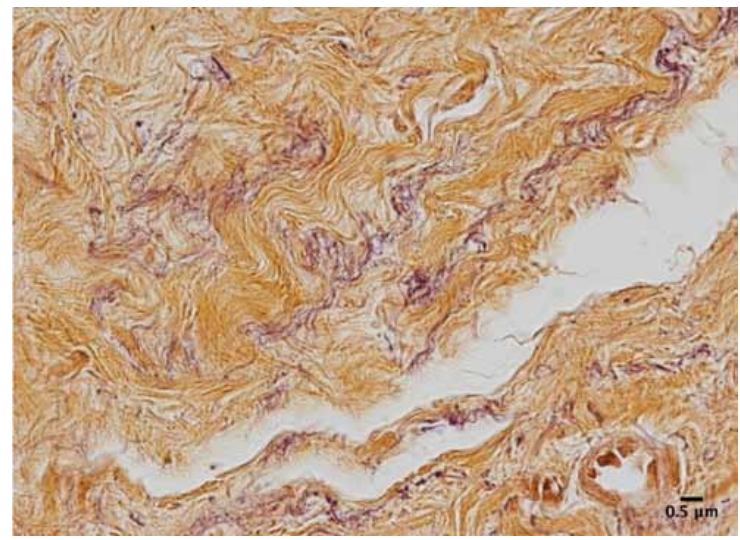

B

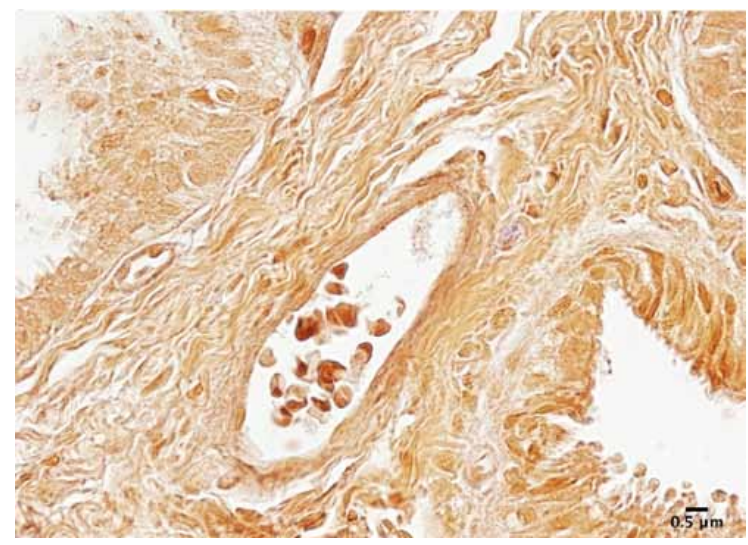

Figure 3 - Testicular appendix vessels. A) Testicular appendix of 2 years-old patient with cryptorchidism. Masson's trichrome X200. B) Testicular appendix of 1 year-old patient in the control group. Masson's trichrome X200. Note the marked increase of vessels in the appendix of the cryptorchidic patient.

A

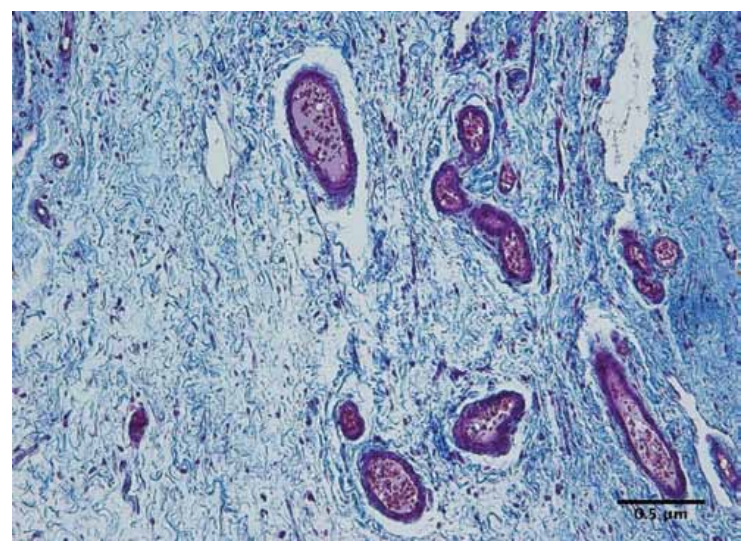

B

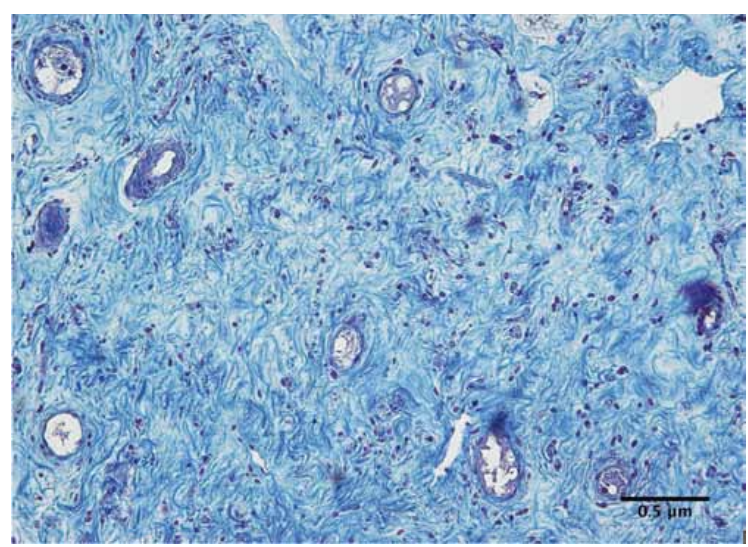


chidism (mean $=4.93 \%)$ in comparison with the control group $($ mean $=9.032 \%)$. Figure- 4 contains photomicrographs comparing the smooth muscle arrangement in the cryptorchidism and the control group appendices.

In the qualitative analysis, type III collagen was observed in both groups, although type III collagen was prevalent in the testicular appendices of patients with cryptorchidism and type I was prevalent in the patients of the control group. Figure- 5 shows examples of the two groups stai- ned with Picro Sirius Red with polarization. The photomicrographs presented a large difference in colors between the groups. This difference can suggest changes in the collagen fiber organization.

\section{DISCUSSION}

Knowledge of the presence, form and location of testicular and epididymal appendices is important in clinical practice because of the possibility of torsion of these structures, their association with

Figure 4 - Distribution of smooth muscle cells of the testicular appendix. A) Testicular appendix of 3-year-old patient with cryptorchidism. Imunohistochemical staining for alfha-actin X200. B) Testicular appendix of 11-year-old patient of the control group. Imunohistochemical staining for alfha-actin X200. Note the marked increase of SMCs in the appendix of the control patient when compared to the cryptorchidic patient.

A

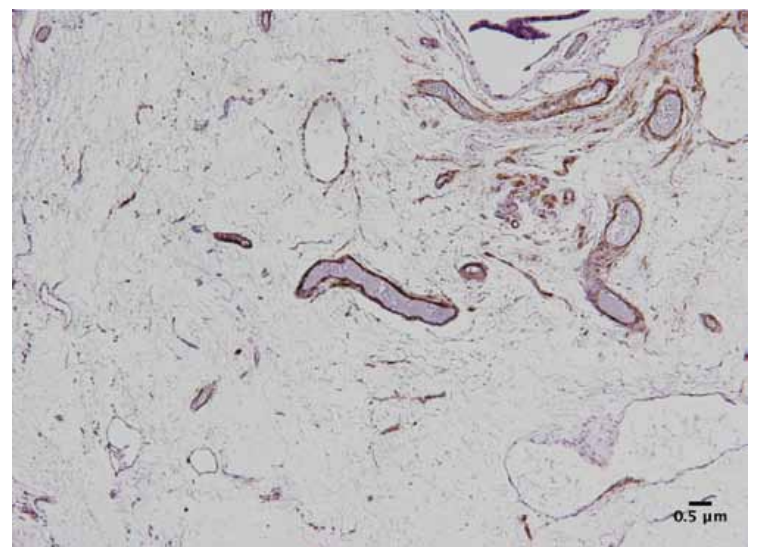

B

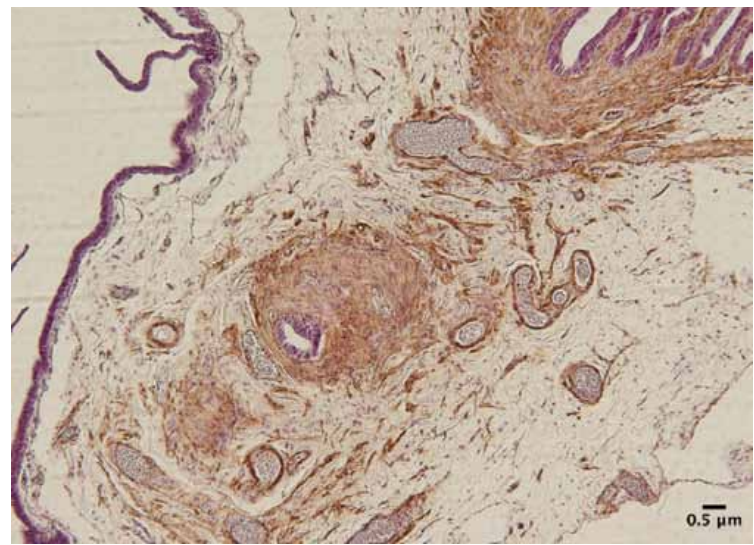

Figure 5-Qualitative collagen distribution in testicular appendices. A) Testicular appendix of 3-year-old patient with cryptorchidism showing prevalence of yellowish color. Picrosirius-polarization method, X400. B) Testicular appendix of 11-years- old patient of the control group showing prevalence of greenish color. Picrosirius- polarization method, X400.

A

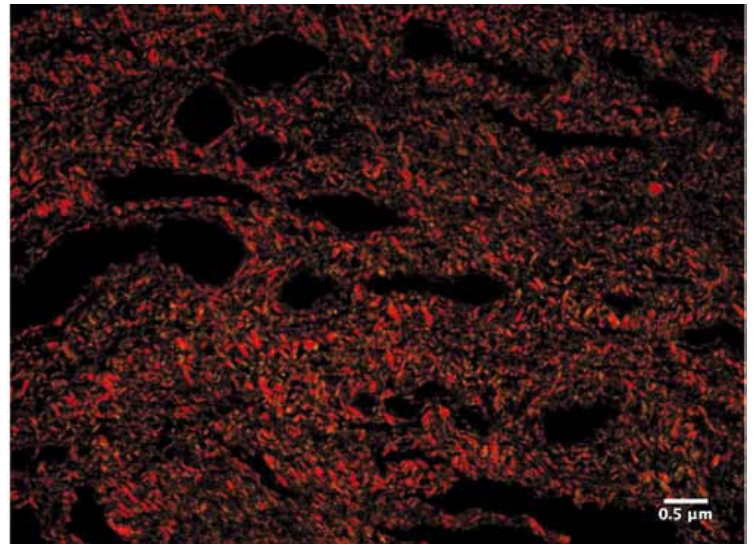

B

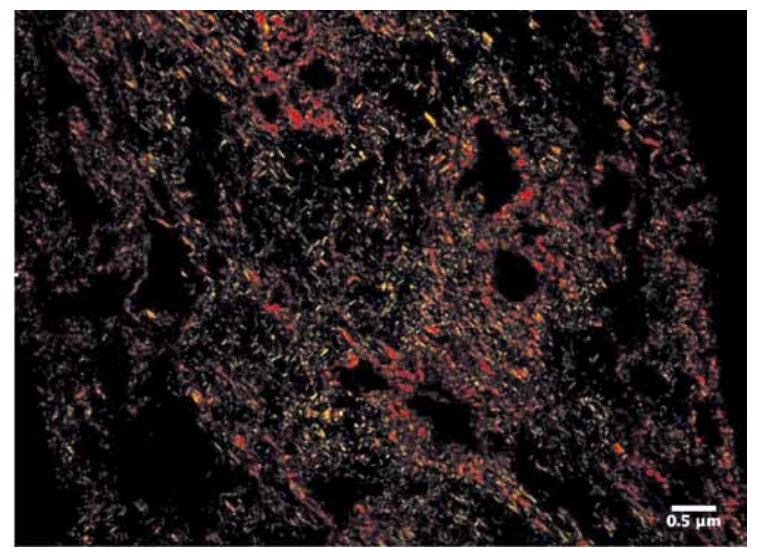


anatomical anomalies and the higher incidence of tumors $(3,15)$. Cryptorchidism is one of the most common congenital anomalies among males, with a rate between 2 and 5\% of full-term births, a rate that can reach $30 \%$ in premature babies (16-18). There are few studies in the literature on the incidence and analysis of the structure of testicular appendices in patients with cryptorchidism and their relation with epididymal anomalies and patency of the vaginal process (11).

Previous studies have shown that testicular appendices occurs significantly less often in patients with cryptorchidism. This can indicate a possible role of testicular appendices in the testicular migration process (19). In the present study, 61.1\% of the testes with cryptorchidism presented testicular appendices, a much higher rate than the 24\% reported by Jozsa (19). We did not find a significant difference in the number of appendices in the testis with cryptorchidism in relation to those of the control group, and also did not find a significant difference in the incidence of appendices in relation to the testicular position in the patients with cryptorchidism.

Cryptorchidism can be associated with various anatomical anomalies, but epididymal anomalies and patency of the vaginal process are among the most frequent (20-22). Epididymal anomalies are associated with cryptorchidism, with prevalence that ranges from 36 to $79 \%(20,21)$. In this study we found $27.7 \%$ of the testes with cryptorchidism also had epididymal anomalies and 56.9\% had patency of the vaginal process. Of the 44 testes with appendices, $68.18 \%$ had patency of the vaginal process and 25\% presented epididymal anomalies. There was no significant difference between the incidence of epididymal anomalies or patency of the vaginal process in the patients with cryptorchidism with and without appendices.

Previous studies have shown that the testicular appendices of patients with cryptorchidism show significant alterations in the estrogen and androgen receptors $(19,23)$. Patients with hydroceles also present alteration in the expression of androgen receptors and epithelial destruction in the testicular appendices (19). These alterations are argued to be associated with increased hydrostatic pressure in the scrotum of patients with hydroceles $(19,23)$.
Reports of structural and ultrastructural changes in the testicular appendices of patients with cryptorchidism are rare $(10,19)$. In this study we did not observe any alterations in the epithelium of the testicular and epididymal appendices between the patients with cryptorchidism and those in the control group, where the majority of cases were made up of patients with hydroceles. We found a large quantity of veins and connective tissue in both the testicular and epididymal appendices. These findings are in agreement with those of Sahni et al. (10) and can confirm the theory that the epithelial layer of the appendices along with the subepithelial capillaries and lymphatic vessels form a functional unit $(8,19)$.

In our samples, we observed the presence of elastic fibers in the appendices, more so in those of patients with cryptorchidism. The increased synthesis of elastic fibers may be associated with excessive distension of an organ (24). We also observed a decrease in the quantity of smooth muscle fibers and predominance of type III collagen in the patients with cryptorchidism. We can speculate that the smooth muscle fiber reduction is the primary event in fibrotic tissue formation. This event is probably correlated to a hydrodistension process. It has been variously shown that a shift towards greenish color in the Picrosirius polarization method is associated with less organized and/or degraded collagen (25). This color change can also occur in earlier phases of the remodeling and repair of connective tissues, when the synthesis of type III collagen is enhanced. Thus, based on these findings, the results of the Picrosirius-polarization method suggest that collagen matrix at the testicular appendices in patients with cryptorchidism is disrupted or degraded, rather than fibrotic, which is consistent with higher hydrostatic pressure. These findings lead us to suggest that in testes without complete migration, testicular appendices may result from the higher intra- abdominal pressure to which these testes are exposed.

In conclusion, we did not find a difference in the incidence of testicular appendices in relation to the testicular position in the patients with cryptorchidism. There also was no increased incidence of anatomical anomalies associated with the testes containing appendices. The testicular appendices showed a significant structural alteration in the pa- 
tients with cryptorchidism: although the epithelium was not changed, the testicular appendices of the patients with cryptorchidism had a larger quantity of elastic fibers and smaller quantity of smooth muscle cells and predominance of type III collagen, remodeling in patients with cryptorchidism.

\section{ACKNOWLEDGEMENT}

By grants from the National Council for Scientific and Technological Development (CNPQ Brazil) and the Rio de Janeiro State Research Foundation (FAPERJ), RJ, Brazil

\section{CONFLICT OF INTEREST}

None declared.

\section{REFERENCES}

1. Johansen TE: Anatomy of the testis and epididymis in cryptorchidism. Andrologia. 1987; 19: 565-9.

2. Nöske HD, Kraus SW, Altinkilic BM, Weidner W: Historical milestones regarding torsion of the scrotal organs. J Urol. 1998; 159: 13-6.

3. Rolnick D, Kawanoue S, Szanto P, Bush IM: Anatomical incidence of testicular appendages. J Urol. 1968; 100: 755-6.

4. Vermeulen CW, Hagerty CS: Torsion of the appendix testis (hydatid of Morgagni) report of two cases with a study of the microscopic anatomy. J Urol. 1945; 54: 459-65.

5. Krukowski ZH, Auld CD: Torsion of the appendix epididymis in a maldescended testis. Br J Urol. 1983; 55: 244-5.

6. Grosfeld JL, O'neil JA, Coran AG, Fonkalsrud EW: Undescended Testis, Torsion and Varicocele; In: Pediatric Surgery 6th (ed.), Chapter 75. Mosby. 2006; pp. 1193-214.

7. Józsa T, Csízy I, Kutasy B, Cserni T, Flaskó T: Decreased incidence of appendix testis in cryptorchidism with intraoperative survey. Urol Int. 2008; 80: 317-20.

8. Posinovec J: Is the unstalked hydatid a functioning organ? Verh Anat Ges. 1969; 63: 751-9.

9. Jacob M, Barteczko K: Contribution to the origin and development of the appendices of the testis and epididymis in humans. Anat Embryol (Berl). 2005; 209: 287-302.

10. Sahni D, Jit I, Joshi K, Sanjeev: Incidence and structure of the appendices of the testis and epididymis. J Anat. 1996; 189: 341-8.

11. Favorito LA, Cavalcante AG, Babinski MA: Study on the incidence of testicular and epididymal appendages in patients with cryptorchidism. Int Braz J Urol. 2004; 30: 49-52.
12. Turek PJ, Ewalt DH, Snyder HM 3rd, Duckett JW: Normal epididymal anatomy in boys. J Urol. 1994; 151: 726-7.

13. Favorito LA, Sampaio FJ: Anatomical relationships between testis and epididymis during the fetal period in humans (10-36 weeks postconception). Eur Urol. 1998; 33: 121-3.

14. Mandarim-de-Lacerda CA, Fernandes-Santos C, Aguila MB: Image analysis and quantitative morphology. Methods Mol Biol. 2010; 611: 211-25.

15. Sundarasivarao D: The Müllerian vestiges and benign epithelial tumours of the epididymis. J Pathol Bacteriol. 1953; 66: 417-32.

16. Kogan S, Hadziselimovic F, Howards SS, Snyder III HM, Huff D: Pediatric andrology. In: Adult and pediatric urology, 3rd (ed.), St Louis. Mosby Year Book. 1996.

17. Gill B, Kogan S: Cryptorchidism. Current concepts. Pediatr Clin North Am. 1997; 44: 1211-27.

18. Cendron M, Huff DS, Keating MA, Snyder HM 3rd, Duckett JW: Anatomical, morphological and volumetric analysis: a review of 759 cases of testicular maldescent. J Urol. 1993; 149: $570-3$.

19. Józsa T, Dienes B, Telek A, Hargitai Z, Pór A, Kiss C: Differential expression of androgen and estrogen receptor of appendix testis in patients with descended and undescended testes. Int J Urol. 2008; 15: 171-4.

20. Marshall FF: Anomalies associated with cryptorchidism. Urol Clin North Am. 1982; 9: 339-47.

21. Mollaeian M, Mehrabi V, Elahi B: Significance of epididymal and ductal anomalies associated with undescended testis: study in 652 cases. Urology. 1994; 43: 857-60.

22. Barthold JS, Redman JF: Association of epididymal anomalies with patent processus vaginalis in hernia, hydrocele and cryptorchidism. J Urol. 1996; 156: 2054-6.

23. Samnakay N, Cohen RJ, Orford J, King PA, Davies RJ: Androgen and oestrogen receptor status of the human appendix testis. Pediatr Surg Int. 2003; 19: 520-4.

24. Cortivo R, Pagano F, Passerini G, Abatangelo G, Castellani I: Elastin and collagen in the normal and obstructed urinary bladder. Br J Urol. 1981; 53: 134-7.

25. Ushiki T: Collagen fibers, reticular fibers and elastic fibers. A comprehensive understanding from a morphological viewpoint. Arch Histol Cytol. 2002; 65: 109-26.

Correspondence address: Dr. Luciano Alves Favorito

Rua Professor Gabizo 104/201

Tijuca, Rio de Janeiro, RJ, 20271-062, Brazil Fax number: + 5521 3872-8802 E-mail: lufavorito@yahoo.com.br 